\title{
The influence of polysorbate tween 80 on somatic health rats in experiment
}

\section{Introduction}

The work of the coagulation/anticoagulation and rheological systems of the body provides movement and circulation of blood, providing its trophic function. ${ }^{1,2}$ Blood is a suspension with the blood cells suspended in it, so the blood has a special structuring. ${ }^{3}$ Changes in the coagulation and rheological systems cause a change in the structuring, retardation of the flow, causes stasis and thrombus of the vessels, and as a consequence, generalized disturbances of the blood flow. One of the main manifestations of blood properties is the dependence of blood viscosity on the diameter of the vessel and blood flow velocity. Among the most important factors determining blood circulation include hematocrit, erythrocyte deformability and their tendency to aggregate, the structure of the blood flow.

Normal structuring of the blood flow is associated with the adequacy of the rheological properties of the blood and the balance of coagulation and anticoagulation.

With all pathological processes, the mechanism of changes in blood flow velocity always works. The effectiveness of blood flow depends on intravascular factors and vascular responses. Vascular reactions, rheological and coagulation properties of blood determine the intensity of microcirculation, which in turn provides adequate needs for blood supply to tissues. ${ }^{3}$

On the one hand, changes in blood circulation support the development of pathology, and on the other hand, the increase and intensification of pathological changes stimulates the process of slowing blood flow. For the normal functioning of systems, organs, tissues, cells, it is necessary to balance all physical and physiological factors that will ensure fluidity of blood corresponding to the needs and loads at a given time.

The daily life of modern people is associated with poorly understood chemical compounds from the point of view of the effect on the blood flow. These are surfactants, in particular polysorbate 80 (trade name: Tween 80, chemical name: polyoxyethylene sorbitan-20monooleate). Tween 80 is used in almost all areas of the industrial sector and daily use. Tween 80 is used in the hygiene industry (cleaning products, toothpaste, lotions, tonics), in the textile industry, in the leather industry, in the paint and varnish industry, in the paper industry, in metallurgy, in agronomy and agriculture, in the food industry, in oil production, in construction, in medicine, in pharmacy. Recently, Tween 80 is used individually as a stabilizer for essential oils, a viscosity regulator, a stabilizer and a dispersing agent. Modern scientific literature on biomedicine does not have data on the effect of surfactants on biological systems. The purpose of our work was the study of Tween- 80 on blood flow in vitro.

\section{Materials and methods}

\section{Animals}

In the experiment, outbred rats participated. In the experiments participated animals that were in the same conditions of nutrition and care: temperature- $22 \mathrm{C}$, humidity-50\%, artificial light-50 lux, normal

\author{
Volume 5 Issue 2 - 2018
}

\author{
Mantskava M, ${ }^{1,2}$ Momtselidze N,, 2 Mitagvaria \\ N,' Davlianidze L,' Devdariani M,' \\ Nebieridze M,' Gumberidze L,' Kvachakidze \\ I,' Sikhariulidze $N^{\prime}$ \\ 'Beritashvili Experimental Centre of Biomedicine, Georgia \\ ${ }^{2}$ Society of Rheology, Georgia
}

Correspondence: Mantskava M, Beritashvili Experimental Centre of Biomedicine, 0I6I, Society of Rheology, 0172, Tbilisi, Georgia, Email mantskavamaka@rambler.ru

Received: November 24, 2017 | Published: April 20, 2018

amount of food and uninterrupted access to water. All rats were divided into a control group-animals that did not receive Tween 80 $(n=5)$ and a group that participated in the experiments-animals that receive Tween $80(n=30)$. The experimental group was divided into 6 subgroups. In each subgroup there were 5 rats. The average weight by subgroups and in control was equal to $183 \pm 27 \mathrm{~g}$. In the control group and in each subgroup there were 3 male animals and 2 female animals.

\section{Microcirculation research}

Determine the influence of Tween 80 on fluidity of blood, we created an original useful model $(n=6)$. For anesthesia, we used chloral hydrate ( $1 \mathrm{ml}$ of $4 \%$ solution per $100 \mathrm{~g}$ of body weight). Anesthesia was done with $0.02 \mathrm{ml}$ of a $2 \%$ solution of promedol per $100 \mathrm{~g}$ of body weight. We isolated the mesentery and placed on a special table with a temperature of $37^{\circ} \mathrm{C}$ under the microscope "Ortoplan, 170/-Plapo $(\times 6.3)$ " with a special chamber Vario-Ortomat "Leitz", Germany. We did alternately the application of Tween 80 to vessels of different diameters, after which they were irrigated with physiological solution.

\section{Rheological research}

In the experiment, 30 individuals of outbred rats participated. Nonnative rats with an average body weight of $183 \pm 27 \mathrm{~g}$ were injected with Tween 80 ( $1 \mathrm{mg}$ per $100 \mathrm{~g}$ of body weight) by injection into the soft tissue of the animal. We took blood from the tail vein. The index of erythrocytes aggregability, which represents aggregated erythrocytes area ratio against whole area of the erythrocytes. Erythrocyte aggregation was evaluated with the recently developed "Georgian technique" providing us with direct and quantitative data. Blood samples were centrifuged and about $0.1 \mathrm{ml}$ blood was diluted 1:200 in own plasma in the Thoma pipettes preliminary rinsed with $5 \%$ sodium citrate solution without addition of any other anticoagulants to the blood under study. Following standard mixing the diluted blood was placed into a glass chamber $0.1 \mathrm{~mm}$ high. The quantitative index of erythrocyte aggregation, which was assessed with a special program at the Texture Analysis System (TAS-plus, "Leitz, Germany), represented itself the relationship of the aggregated and unaggregated red cells. Evaluation of erythrocytes deformability was performed with an aid of the nucleopore membrane filter method, 
which is based on assessing velocity of the erythrocytes passage through the very small pores $(5 \mu \mathrm{m}$, which is a diameter of the smallest capillary) of the filter, at constant pressure ( $10 \mathrm{~cm}$ of water column) and temperature $\left(37^{\circ} \mathrm{C}\right)$. Obtaining the pure erythrocytes was performed by centrifuging the blood sample at $3000 \mathrm{rpm}$, for $15 \mathrm{~min}$. Resulting plasma was aspirated with micropipette and the remaining blood cells were added with bovine serum albumin $(0.2 \mathrm{mg}$ per $5 \mathrm{ml})$ dissolved in the phosphate buffer. Then the blood was centrifuged second time at $1000 \mathrm{rpm}$ for $5 \mathrm{~min}$. The precipitated erythrocytes, as well as thin layer of leukocytes and thrombocytes, were separated from the phosphate buffer. This procedure was repeated three times. Purified erythrocyte mass was diluted in the phosphate buffer, with hematocrit of $10 \%$. Evaluation of the deformability index implied measuring a velocity of the erythrocyte passage through the filter $(\mathrm{mm} / \mathrm{min})$ was recorded. The high quality polycarbonate filters (with $5 \mu \mathrm{m}$ diameter pores) were used in measuring procedures. Blood plasma viscosity was examined in the capillary viscometer at $37^{\circ} \mathrm{C}$. Diameter of the capillary was about $1.8 \mathrm{~mm}$. Displacement of plasma samples was induced by the gravity force related to the difference of niveaux of the plasma under study - about 65. (Without application of an additional pressure) For evaluation of the plasma viscosity in centipoises (cp) we determined the calibration factor $(\mathrm{F})$. Blood plasma viscosity was calculated by multiplying the time for plasma displacement through the capillary by the instrument calibration factor. Evaluation of Systemic Hematocrit: Systemic hematocrit was assessed with centrifuging the blood sample in the standard hematocrit centrifuge, at $8000 \mathrm{rpm}$, for $10 \mathrm{~min}$.

\section{Study of the general picture of blood}

The number of erythrocytes, leukocytes, platelets was made by a clinical hematological analyzer with a control scale for rats (HUMACOUNT, model HUMACOUNT (manufactured by Firm Human GmbH, Germany)

\section{Measuring rectal temperature}

The rectal temperature was measured with an electronic multivoltmeter DT-838 with the attached temperature sensors.

\section{Assessment of the psychoemotional state}

Registration of behavioral components: using the Laboras system (Metris, the Netherlands), which made it possible to calculate the duration of such behaviors as locomotion (horizontal activity), immobility, upright (vertical activity), washing ${ }^{4}$ and estimated the time of unrecognized movements. ${ }^{4,5}$

\section{Description of the preparation}

Tween 80 -Viscous, oily liquid from light yellow to amber, odorless with a density of $1.06-1.09 \mathrm{~g} / \mathrm{ml}$ with a hydrophilic-lipophilic balance of 15-15.6 is readily soluble in vegetable oils. The form of release $-50 \mathrm{ml}$ a bottle, trade mark Aromashka, company Laboratoire RosierDavenne.

\section{Description of the experiments}

Measurements and studies were conducted at different time intervals. The total number of animals we divided into subgroups of five in each. In each subgroup we measured hemorheological indices, total blood composition, rectal temperature and psychoemotional state 12 hours after injection of Tween 80 , through 24 , through 72 , through1 week, through 2 weeks, respectively. The control group consisted of animals that did not receive Tween $80(n=5)$. The average weight of the animals in the control group and in the experimental subgroups corresponded to each other. The analysis of the data was carried out by the statistical programs Origin 4.1. (Microcat.Software. Inc) and Microsoft Excel, the protocol for carrying out the research was in accordance with the European Convention for the Protection of Vertebrates used for Experiments and Other Scientific Purposes (ETS 123) - "Strasburg Convection". ${ }^{6}$ For the experiment was obtained permission of ethical committee of the our organizations, which participated on experiments.

\section{Results and discussion}

The influence of the influence of Tween 80 on the fluidity of blood was examined by us on the mesenteric model of microcirculation. We alternately selected capillaries with 1: with a normal blood flow; 2: with stasis; 3: blind capillaries (plasmatic). We conducted the application of Tween 80 on the visual capillary chosen by us. It turned out, that in all varieties (normal capillary, stasis capillary, plasma capillary) Tween 80 does not affect the capillary current. After Tween 80 the applications, the capillary network was irrigated with physiological saline.

The total blood composition, hemorheological parameters, rectal temperature in the rats in the control group and after Tween 80 injections are shown in Table 1.

Table I Mean values of hemorheological parameters, total blood composition and rectal temperature of experimental specimens after injection of Tween 80 in different subgroups and in controls $M \pm m^{*}$

\begin{tabular}{|c|c|c|c|c|c|c|c|c|c|}
\hline \multirow[t]{2}{*}{ N Subgroup } & \multirow[t]{2}{*}{$\begin{array}{l}\text { Time } \\
\text { intervals }\end{array}$} & \multicolumn{4}{|c|}{ Hemorheological parameters } & \multicolumn{3}{|c|}{ General composition of blood } & \multirow{2}{*}{$\begin{array}{l}\text { Rectal } \\
\text { temperature, } \\
{ }^{\circ} \mathrm{C}\end{array}$} \\
\hline & & EAI, \% & EDI,\% & Viscosity, sP & Hct, gr/l & RBC, $10 \mid 2 / I$ & $\begin{array}{l}\text { WBC, } \\
109 / 1\end{array}$ & $\begin{array}{l}\text { Platelets, } \\
\text { 109/I }\end{array}$ & \\
\hline 1 & $\begin{array}{l}\text { through } 12 \\
\text { hours }\end{array}$ & $20 \pm 3,4$ & $2,25 \pm 0,03$ & $1,30 \pm 0,05$ & $195 \pm 12$ & $10,4 \pm 1,2$ & $14,2 \pm 0,9$ & $320 \pm 27$ & $38,3 \pm 0,7$ \\
\hline II & $\begin{array}{l}\text { through } 24 \\
\text { hours }\end{array}$ & $19 \pm 3,2$ & $2,25 \pm 0,04$ & $1,50 \pm 0,05$ & $190 \pm 14$ & $10,6 \pm 2,0$ & $15,7 \pm 1,2$ & $540 \pm 54$ & $37,3 \pm 0,9$ \\
\hline III & $\begin{array}{l}\text { through } 72 \\
\text { hours }\end{array}$ & $20 \pm 4,5$ & $2,25 \pm 0,03$ & $1,25 \pm 0,05$ & $140 \pm 8$ & $6,5 \pm 2,4$ & $17,2 \pm 1,8$ & $480 \pm 59$ & $38,8 \pm 0,5$ \\
\hline IV & $\begin{array}{l}\text { through I } \\
\text { week }\end{array}$ & $19 \pm 3,2$ & $2,25 \pm 0,03$ & $1,25 \pm 0,05$ & $140 \pm 7$ & $6,8 \pm 3,5$ & $15,9 \pm 1,9$ & $280 \pm 70$ & $38,2 \pm 0,7$ \\
\hline V & $\begin{array}{l}\text { through } 2 \\
\text { weeks }\end{array}$ & $20 \pm 2,8$ & $2,25 \pm 0,03$ & $\mathrm{I}, 30 \pm 0,05$ & $165 \pm 4$ & $5,9 \pm 2,0$ & $16,5 \pm 1,0$ & $300 \pm 35$ & $38,5 \pm 0,8$ \\
\hline VI & control & $20 \pm 2,5$ & $2,25 \pm 0,03$ & $1,25 \pm 0,05$ & $160 \pm 6$ & $6,6 \pm 2,2$ & $15,2 \pm 1,0$ & $330 \pm 20$ & $38 \pm 0,9$ \\
\hline
\end{tabular}

*Note In the table are the parameters that together with the psychoemotional state provide somatic health. All parameters averaged over the subgroups and in the control group 
From the biophysical point of view, blood is a heterogeneous, multicomponent system of corpuscular nature, suspension of the elemental elements in a colloidal solution of proteins, lipids and electrolytes, which is blood plasma. The perfusion of tissues is ensured by the passage of this concentrated suspension of solid particles through a system of microvessels, whose diameter in some areas is smaller than the diameter of the particles themselves. The features of the elastic-rigid properties of blood, like suspensions are manifested at the level of microcirculation. ${ }^{7}$ Therefore, the definition of the rheological properties of blood and microcirculation gives an unambiguous answer about blood circulation as a whole. ${ }^{3,7}$ At the rectal temperature, a general blood test and an assessment of the psycho-emotional state, one can judge the somatic health of the test subjects.

This paper describes original methods for studying the effects of Tween 80 on the rheological parameters of blood, on the total blood composition, on rectal temperature and behavioral tests, and on the effect of Tween 80 directly on microcirculation in vivo. According to our data, it turned out that Tween 80 does not affect the total blood composition and haemorheological properties of blood, except for the aggregation capacity of red blood cells and their counts. However, this increase is normalized through 72 hours after the injection of Tween 80 into the muscle then remains within normal limits. Background of these processes is unchanged leukocyte count, unremoved rectal temperature, background of normal behavioral tests. All this indicates the absence of inflammation.

Temporary increase in erythrocyte quantities can be attributed to the components of the compensatory mechanism, which develops during oxygen starvation. ${ }^{8}$

Microcirculation in experiments in vivo was not affected by the direct influence of Tween 80 on capillaries. This indicates the inactivity of Tween 80 on the permeability of blood vessels, the erythrocyte membrane, and the pressure gradient. Tween 80 acts on the person contact through the skin and in pairs of inhaled air. Our tried and tested technique is injection with a drug. Such an approach is justified scientifically in connection with the fact that the detection and prevention of adverse effects of a toxic factor on the body is one of the main tasks of preventive toxicology, the main method of which is experimental research methods. ${ }^{5}$ Applying conventional methods of toxicological studies to evaluate detergents and cleaners, toxicologists do not have sufficient opportunities to satisfy the requirements of practice, so there is a need to optimize existing methodological approaches. ${ }^{9}$ Microcirculation in experiments in vivo was not affected by the direct influence of Tween 80 on the capillary. Actual is the search for adequate alternative test systems and their scientific justification for predicting the impact of the ability of surfactants, in particular Tween 80 , on blood fluidity, which is responsible for the adequacy of blood circulation.

\section{Conclusion}

Determining the somatic health of the tested animals under the influence of the polysorbate Twin 80 , with which we stack up in everyday life. Such a study is very important, as to understand the risks associated with the use of polysorbate Twin 80 . It turned out that polysorbate is not a dangerous and harmful substance, but observed short-term changes in parameters that provide somatic health of animals. But since in experiments we injected polysorbate intramuscularly, this is an exaggeration of reality. Therefore, the harmful effect of polysorbate Twin 80 is not significant. In that work the methods of studying the harmful effects of surfactants on blood circulation have been optimized, which provides the value of this study for the development of alternative biological test objects that play a supporting role in the identification of the hazard of potentially harmful substances.

\section{Acknowledgements}

None.

\section{Conflict of interest}

The authors declare no conflict of interest.

\section{References}

1. Kamensky A, Kamkin A. Fundamental and clinical physiology. Moscow, Russia: Academia; 2004.

2. Hoffman M, Monroe D. Coagulation: a modern view of hemostasis. Hematol Oncol Clin North Am. 2007;21(1):1-11.

3. Mchedlishvili G. Basic factors determining the hemorheological disorders in the microcirculation. Clin Hemorheol Microcirc. 2004;30(34):179-180.

4. Karkischenko NN, Gracheva SV. Manual on laboratory animals and alternative models in biomedical technology. Moscow, Russia: Profil2S; 2010:358.

5. Directory of the doctor of veterinary medicine. Belarus; 2006:1496.

6. European Convention for the Protection of Vertebrates used for Experiments and Other Scientific Purposes (ETS 123).

7. Mantskava M, Momtselidze N. Blood hemorheological status. Germany: Lambert Academic Publishing; 2014:78.

8. Mikhailova N, Gorokhova L, Kazitskaya A, et al. Assessment of biochemical changes in peripheral blood in the early stages of experimental fluoride intoxication. Acta Biomedica Scientifica. 2010;4:43-46.

9. Kozhevnikova I, Morylev I. Egorova N. The use of alternative toxicological methods in the practice of the department of preventive toxicology, Abstracts. Second congress of Russian toxicologists. 2003:131-132. 